\title{
Les composantes du rayonnement gamma et du rayonnement cosmique dans les habitations. Évaluation par spectrométrie
}

\author{
J. BERENS-TOMCZYŃSKA et J. PEŃSKO (*) \\ (Manuscrit reçu le 28 mars 1980)
}

\begin{abstract}
RÉSUMÉ
On a mis au point une méthode spectrométrique de mesure de débits de dose dans les locaux à usage d'habitation. Pour la réalisation des mesures in situ, on a utilisé un spectromètre gamma (avec un cristal de $\mathrm{NaI} / \mathrm{Tl}$ ), et un analyseur d'amplitude multicanal. Les mesures effectuées dans quatre villes de Pologne tiennent compte de la technologie utilisée pour chaque type de construction. Pour évaluer la composante ionisante des rayons cosmiques à l'intérieur des constructions, on a fait des mesures à l'aide d'une chambre d'ionisation à haute pression. L'erreur des mesures effectuées avec le spectromètre gamma ne dépasse pas $\pm 20 \mathrm{p}$. cent.
\end{abstract}

\begin{abstract}
A spectrometric method of dose rate measurement inside dwelling-houses has been developed using a gamma ray spectrometer with a $3 \times 4$ in. NaI/Tl crystal and a multichannel pulse-height analyzer. The in situ measurements were carried out in four cities in Poland taking into account the building technology indicated by the Research Center for Light Concrete Technology in Warszawa. In order to estimate the ionizing component of cosmic rays inside the buildings, the measurements were performed with a high-pressure ionization chamber. The error on the measurements made with the gamma ray spectrometer did not exceed \pm 20 p. cent.
\end{abstract}

\section{INTRODUCTION}

L'utilisation de nouveaux matériaux de construction à base de déchets des industries chimiques et énergétiques est de plus en plus répandue depuis quelques années en Pologne. Certains de ces produits bruts tels que les scories de hauts-fourneaux ou de chaudières, les cendres volantes et le gypse,

(*) Institut de Recherche nucléaire, Département de Protection contre les Rayonnements, 05-400 Swierk/Otwock, Pologne.

RADIOPROTECTION, VOL. 15 - 0033-8451/1980/193/\$ 5.00/C) Bordas-Dunod 
en tant que produit dérivé, contiennent des quantités importantes de radionucléides naturels. L'évaluation des doses de rayonnements gamma à l'intérieur des habitations et la comparaison avec les doses que l'on rencontre en plein air est donc devenue une tâche importante, puisque les hommes passent près de 75 p. cent de leur temps à l'intérieur des habitations.

La littérature fournit peu de résultats de mesures d'irradiation externe à l'intérieur des immeubles. C'est à HultQvist que l'on doit l'une des études les plus détaillées [4]. L'auteur a mesuré l'ionisation de l'air sous l'effet du rayonnement gamma à l'intérieur de trois types d'habitations : en bois, en brique et en béton léger. PEŃsko et al. ont donné des résultats de mesures de débits de dose de rayonnements gamma à l'intérieur de 97 logements récemment construits en béton, en grave-ciment, en gazosilicate cellulaire, en bétoncendres et en briques [8]. OHLSEN a mené d'importantes recherches sur ce sujet [7]; il a relevé les débits de dose de rayonnement gamma en plein air (1 097 mesures) et à l'intérieur (667 mesures) en différents endroits de l'Allemagne de l'Est. Un travail similaire a été récemment effectué en République démocratique allemande et en Australie [5, 10].

Pour la plupart de ces mesures, on a utilisé des dosimètres portatifs avec chambre d'ionisation à haute pression. La méthode est rapide et précise, mais elle ne tient pas compte des différents types de rayonnements provenant des diverses composantes de l'irradiation naturelle. La méthode spectrométrique in situ permet une évaluation quantitative de l'influence de chaque radionucléide pris séparément sur le débit de dose total à l'intérieur des immeubles.

\section{2. ÉVALUATION DU DÉBIT DE DOSE DANS LES LOCAUX A USAGE D'HABITATION}

Le rayonnement gamma à l'intérieur des immeubles est dû à ${ }^{40} \mathrm{~K}$ et aux chaînes de ${ }^{238} \mathrm{U}$ et ${ }^{232} \mathrm{Th}$. Chacun de ces émetteurs ou chacune de ces chaînes peut être caractérisé par un seul pic d'absorption totale. Ainsi donc, la chaîne de ${ }^{238} \mathrm{U}$ peut être représentée par le pic de $1,76 \mathrm{MeV}$ de ${ }^{214} \mathrm{Bi}$, la famille de ${ }^{232} \mathrm{Th}$ par le pic de 2,62 $\mathrm{MeV}$ de ${ }^{208} \mathrm{Tl}$ et, enfin, le pic de $1,46 \mathrm{MeV}$ représente ${ }^{40} \mathrm{~K}$.

Le débit de dose absorbée dans l'air, au centre d'une pièce standard, dû à des photons d'énergie $E_{i}$, et englobant la contribution du rayonnement diffusé et rétrodiffusé, est donné par l'équation [2] :

$$
\dot{D}_{i}=505 \cdot 10^{-2} \varphi_{i} \cdot E_{i} \cdot(\mu / \rho)_{i} \cdot B_{i} \cdot\left(1+A_{\varphi_{i}}\right) \quad(\text { en } \mathrm{mG} / \mathrm{an})
$$

où $\varphi_{i}$ représente la densité de flux $\left(\mathrm{en}^{-2} \mathrm{~cm}^{-2} \cdot \mathrm{s}^{-1}\right) ;(\mu / \rho)_{i}$ le coefficient d'atténuation massique pour des photons d'énergie $E_{i}$ dans l'air $\left(\mathrm{en}^{\mathrm{cm}} \mathrm{-}^{-2} \cdot \mathrm{s}^{-1}\right)$; $B_{i}$ le facteur d'accumulation pour une activité uniformément répartie dans le matériau constituant le mur; $A_{\varphi,}$ l'albedo de l'exposition. 


\subsection{ESTIMATION DE LA DENSITÉ DE FLUX}

On peut obtenir la densité de flux $\varphi_{i}$ à partir de la surface nette du pic d'absorption totale et du rendement du détecteur selon la formule

$$
\varphi_{i}=\frac{\text { nombre de coups à l'intérieur du pic }}{\text { rendement du détecteur }} .
$$

On obtient les spectres d'impulsion au moyen d'un cristal NaI/Tl $(3 \times 4$ in.) protégé uniquement par un mince conteneur en aluminium. Le cristal est couplé optiquement à un EMI 9530 (tube photomultiplicateur) raccordé lui-même à un analyseur d'amplitude 800 canaux portatif du type Nokia.

L'étalonnage a été réalisé en laboratoire à l'aide de mesures de spectres gamma émis par des sources ponctuelles convenablement choisies d'intensité connue. Le rendement absolu du détecteur est donné par le nombre de coups par seconde dans le pic d'absorption totale pour une densité de flux de $1 \mathrm{~cm}^{-2} \cdot \mathrm{s}^{-1}$. Les sources sont placées dans l'axe de symétrie du cristal et on fait varier la distance séparant le cristal de la source afin d'obtenir différentes valeurs du flux incident. Toutes les mesures sont faites sans que l'on déduise le bruit de fond afin d'avoir un continuum semblable aux conditions in situ.

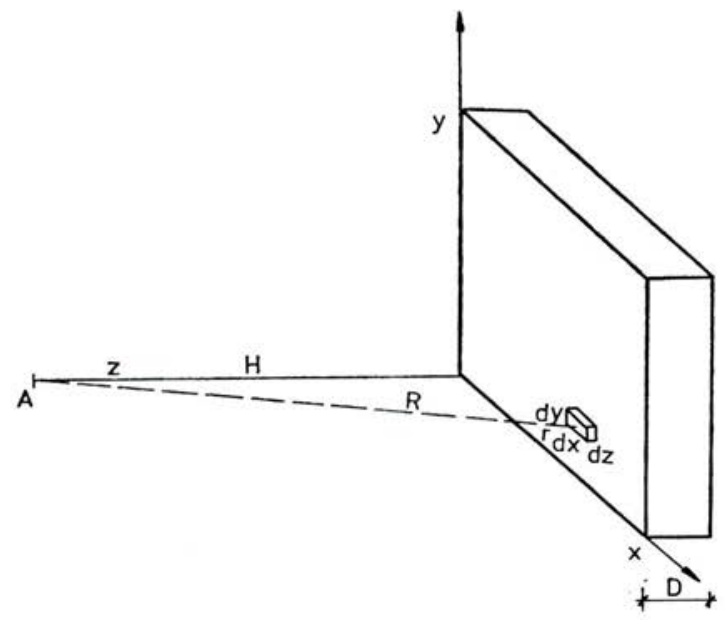

FIg. 1. - Modèle schématique de calcul des facteurs d'accumulation.

\subsection{Calcul des facteurs d'accumulation}

Le facteur d'accumulation pour des émetteurs gamma uniformément répartis dans l'ensemble des murs détermine la contribution du rayonnement diffusé au débit de dose absorbée dans l'air. 
Pour une énergie donnée de rayonnement gamma émis par des radionucléides uniformément répartis dans une plaque d'un matériau donné, le facteur d'accumulation du débit de dose $B$ dépend de $X, Y, D$, qui représentent les dimensions de la plaque, ainsi que de $H$ qui représente la distance entre la plaque et le point pour lequel on détermine le facteur d'accumulation. La figure 1 représente la géométrie particulière qui a été choisie dans cette étude.

Les valeurs suivantes sont définies pour le point $A$ :

1. Débit de dose $P_{1}$ dû aux photons primaires

$$
P_{1}=K \int_{H}^{H+D} \int_{0}^{Y} \int_{0}^{X} \frac{1}{R^{2}} e^{-\mu r} d x d y d z,
$$

où $R$ représente la distance du point $A$ à l'élément de volume $d v=d x d y d z$; $r$ représente la distance partielle du bord de la plaque à l'élément de volume $d v$; $\mu$ représente le coefficient d'atténuation qui dépend de l'énergie des rayons gamma et de la nature de la plaque et $K$ est une constante. défini par

2. Le débit de dose des photons primaires et secondaires diffusés est

$$
P_{2}=K \int_{H}^{H+D} \int_{0}^{Y} \int_{0}^{X} \frac{1}{R^{2}} e^{-\mu r} B(r) d x d y d z,
$$

où $B(r)$ représente le facteur d'accumulation pour une source ponctuelle.

Le facteur d'accumulation $B$ pour une activité uniformément répartie dans la plaque rectangulaire est donné par

$$
B=\frac{P_{2}}{P_{1}}=\frac{\int_{H}^{H+D} \int_{0}^{Y} \int_{0}^{X}\left(1 / R^{2}\right) e^{-\mu r} B(r) d x d y d z}{\int_{H}^{H+D} \int_{0}^{Y} \int_{0}^{X}\left(1 / R^{2}\right) e^{-\mu r} d x d y d z} .
$$

BERGER et al. et TAYLOR indiquent les facteurs d'accumulation pour une source ponctuelle $[3,9]$.

Les valeurs extrêmes du facteur d'accumulation obtenues pour différentes énergies des rayonnements gamma sont données ci-dessous :

\begin{tabular}{|c|c|}
\hline $\begin{array}{c}\text { Énergie } \\
(\mathrm{MeV})\end{array}$ & Valeurs extrêmes de B \\
\cline { 1 - 2 } 1,46 & $1,31-2,03$ \\
1,76 & $1,24-1,86$ \\
2,62 & $1,15-1,59$ \\
\hline
\end{tabular}




\subsection{Albedo De L'EXPosition}

On définit l'albedo d'exposition comme étant l'accroissement relatif du débit d'exposition produit par le rayonnement rétrodiffusé. Dans le présent article, les calculs de débits de dose sont effectués en utilisant les valeurs de l'albedo d'exposition pour une incidence isotope des photons gamma sur une paroi semi-infinie tirées de LEIDMÖRFER [6] :

\begin{tabular}{|c|c|}
\hline $\begin{array}{c}\text { Énergie } \\
(\mathrm{MeV})\end{array}$ & Albedo d'exposition \\
\cline { 1 - 1 } 1 & 0,186 \\
2 & 0,0868 \\
4 & 0,0630 \\
\hline
\end{tabular}

Les valeurs d'albedo retenues ne dépendent pas de la géométrie des parois puisque, dans le cas qui nous intéresse, les distances de mur à mur à l'intérieur de la pièce considérée sont supérieures à $2 \mathrm{~m}$ et les valeurs de $\mu D$ sont supérieures à 1 . Selon les données fournies par la littérature [1] l'influence de la géométrie des murs sur l'albedo d'exposition est inférieure à 3 p. cent dans ce type de cas.

\section{RÉSULTATS DES MESURES ET CONCLUSIONS}

Des mesures de débits de dose dans des locaux à usage d'habitation ont été effectuées dans quatre villes de Pologne; ces mesures tenaient compte de la technologie utilisée pour chaque type de construction telle qu'elle est indiquée par le Centre de recherches sur la technologie du béton léger de Varsovie. Il s'agissait de trois maisons individuelles à Pulawy, de deux autres à Ostroleka, de cinq appartements dans une tour à Czestochowa et de onze appartements dans le district de Varsovie-Ursynów.

On a effectué 20 séries de mesures. Dans les immeubles, on a fait les mesures à plusieurs étages différents; le détecteur était placé au centre de la pièce.

En même temps, des mesures ont été effectuées à l'aide d'une chambre d'ionisation remplie d'argon sous une pression de 30 atmosphères. La chambre est constituée par une enceinte en acier de 51 de volume et de $4 \mathrm{~mm}$ d'épaisseur de paroi. La différence entre les résultats obtenus par ces deux méthodes traduit la présence de la composante cosmique.

On a également effectué une série de mesures en plein air à l'aide d'une chambre d'ionisation à haute pression.

Ce travail fait la preuve de la faisabilité et de l'utilité de l'application de méthodes simples d'analyses de spectres à des mesures spectrométriques de rayonnements gamma obtenues directement dans les locaux d'habitation au moyen d'un grand cristal de $\mathrm{NaI} / \mathrm{Tl}$. Ces mesures peuvent être réalisées 
TABLEAU I

RÉSULTATS DES MESURES SPECTROMÉTRIQUES DE DÉBITS DE DOSE DANS LES HABITATIONS

\begin{tabular}{|c|c|c|c|c|c|c|}
\hline \multirow{2}{*}{$\begin{array}{c}\text { Situation } \\
\text { géographique }\end{array}$} & \multirow{2}{*}{$\begin{array}{l}\text { Numéro } \\
\text { de la } \\
\text { mesure }\end{array}$} & \multicolumn{4}{|c|}{ Débit de dose (mGy/an) } & \multirow{2}{*}{ Remarques } \\
\hline & & $\mathbf{K}$ & $\mathbf{U}$ & Th & Total & \\
\hline \multirow{3}{*}{$\begin{array}{l}\text { Pulawy, maisons } \\
\text { individuelles }\end{array}$} & 1 & 0,30 & 0,017 & 0,035 & 0,35 & \multirow{4}{*}{$\begin{array}{l}\text { Béton léger } \\
\text { avec sable; } \\
\text { en plafond } \\
\text { béton ordinaire }\end{array}$} \\
\hline & 2 & 0,29 & 0,017 & 0,026 & 0,34 & \\
\hline & 3 & 0,33 & 0,026 & 0,026 & 0,39 & \\
\hline \multicolumn{2}{|c|}{ Moyenne............. } & 0,30 & 0,020 & 0,029 & 0,36 & \\
\hline \multirow{4}{*}{ Czestochowa } & 1 & 0,23 & 0,035 & 0,052 & 0,31 & \multirow{5}{*}{$\begin{array}{c}\text { Trois murs } \\
\text { en béton ordinaire, } \\
\text { un mur } \\
\text { en béton léger } \\
\text { avec cendres volantes }\end{array}$} \\
\hline & 2 & 0,23 & 0,035 & 0,043 & 0,30 & \\
\hline & 3 & 0,25 & 0,035 & 0,061 & 0,35 & \\
\hline & 4 & 0,26 & 0,043 & 0,043 & 0,32 & \\
\hline \multicolumn{2}{|c|}{ Moyenne............. } & 0,24 & 0,037 & 0,049 & 0,32 & \\
\hline Ostrolęka, mai- & 1 & 0,56 & 0,017 & 0,20 & 0,78 & \multirow{3}{*}{$\begin{array}{c}\text { Béton léger } \\
\text { avec cendres volantes }\end{array}$} \\
\hline sons individuelles & 1 & 0,54 & 0,017 & 0,20 & 0,78 & \\
\hline \multicolumn{2}{|c|}{ Moyenne...$\ldots \ldots \ldots$} & 0,55 & 0,017 & 0,21 & 0,78 & \\
\hline \multirow{6}{*}{$\begin{array}{l}\text { Varsovie- } \\
\text { Ursynów, } \\
\text { Stoklosy }\end{array}$} & 1 & 0,51 & 0,017 & 0,10 & 0,63 & \multirow{7}{*}{ Béton ordinaire } \\
\hline & 2 & 0,62 & 0,017 & 0,08 & 0,72 & \\
\hline & 3 & 0,49 & 0,017 & 0,08 & 0,58 & \\
\hline & 4 & 0,61 & 0,026 & 0,08 & 0,72 & \\
\hline & 5 & 0,63 & 0,026 & 0,08 & 0,73 & \\
\hline & 6 & 0,51 & 0,026 & 0,10 & 0,64 & \\
\hline \multicolumn{2}{|c|}{ Moyenne............. } & 0,56 & 0,022 & 0,09 & 0,67 & \\
\hline & 1 & 0,64 & 0,026 & 0,09 & 0,76 & \multirow{6}{*}{$\begin{array}{c}\text { Béton " keramzyt " } \\
\text { (béton léger avec } \\
\text { agrégat artificiel) }\end{array}$} \\
\hline Varsovie- & 2 & 0,54 & 0,017 & 0,06 & 0,65 & \\
\hline Ursynów, & 3 & 0,67 & 0,017 & 0,04 & 0,73 & \\
\hline \multirow[t]{2}{*}{ Koński Jar } & 4 & 0,51 & 0,009 & 0,04 & 0,56 & \\
\hline & 5 & 0,59 & 0,026 & 0,04 & 0,66 & \\
\hline \multicolumn{2}{|c|}{ Moyenne...$\ldots \ldots \ldots$} & 0,59 & 0,019 & 0,05 & 0,66 & \\
\hline
\end{tabular}

très facilement sans modification particulière des détecteurs et des analyseurs d'amplitude que l'on trouve dans la plupart des laboratoires nucléaires. L'application de ces méthodes suppose que l'on dispose de sources radioactives étalonnées et de certaines expérimentations en laboratoires pour la détermination de la réponse du détecteur. 


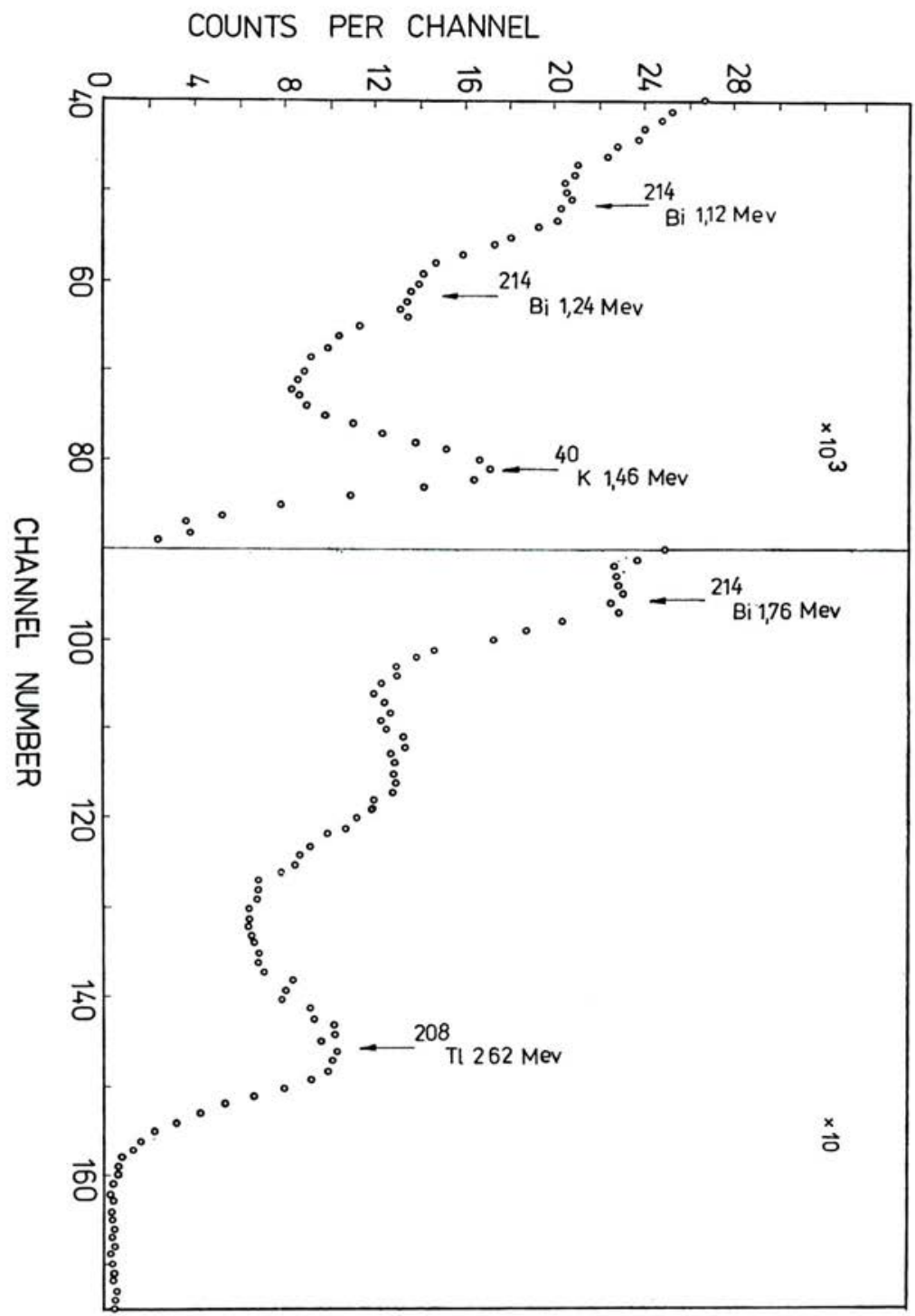

FIG. 2. - Spectre de rayonnement gamma obtenu dans un appartement à Varsovie Ursynów.

Le tableau I résume les mesures spectrométriques. Les résultats montrent que, dans les locaux étudiés, ${ }^{40} \mathrm{~K}$ est responsable de la principale composante du rayonnement gamma. Les valeurs de débit de dose pour ce radionucléide sont comprises entre 0,30 et $0,67 \mathrm{mGy} / \mathrm{an}$; les résultats ci-dessus incluent 


\section{TABLEAU II}

Comparaison des valeurs MOYENNES de déBit de dose (mGy/an) OBTENUES PAR SPECTROMÉTRIE ET PAR CHAMBRE D'IONISATION

\begin{tabular}{|c|c|c|c|c|}
\hline $\begin{array}{c}\text { Situation } \\
\text { géographique }\end{array}$ & $\begin{array}{c}\text { Spectro- } \\
\text { métrie }\end{array}$ & $\begin{array}{c}\text { Chambre } \\
\text { d'ionisation }\end{array}$ & $\begin{array}{c}\text { Rayonnement } \\
\text { cosmique }\end{array}$ & $\begin{array}{c}\text { Mesures } \\
\text { en plein air }\end{array}$ \\
\cline { 1 - 2 } Pulawy ............. & 0,36 & 0,73 & 0,37 & 0,70 \\
Czestochowa....... & 0,32 & 0,62 & 0,30 & 0,60 \\
Ostroleka.......... & 0,78 & 1,08 & 0,30 & 0,83 \\
Varsovie-Stoklosy... & 0,67 & 0,98 & 0,31 & 0,68 \\
Varsovie-Konski Jar. & 0,66 & 0,98 & 0,32 & 0,68 \\
\hline
\end{tabular}

également une contribution de ${ }^{40} \mathrm{~K}$ provenant du tube photomultiplicateur. La contribution de ${ }^{232} \mathrm{Th}$ avec des valeurs de débit de dose comprises entre 0,03 et $0,22 \mathrm{mGy} / \mathrm{an}$ est bien plus faible. Le débit de dose le plus faible $(0,017-0,043 \mathrm{mGy} / \mathrm{an})$ provient $\mathrm{de}^{238} \mathrm{U}$.

La méthode spectrométrique apporte des renseignements importants sur les composantes du rayonnement gamma dans les locaux d'habitation. La comparaison de la méthode spectrométrique et de la méthode avec chambre d'ionisation permet d'évaluer la contribution du rayonnement cosmique (tableau II). Les différences observées entre les doses moyennes mesurées à l'intérieur et en plein air sont importantes dans le cas des constructions faites en béton léger avec cendres volantes (la dose à l'intérieur étant supérieure de 30 p. cent) et dans le cas des constructions en ciment ordinaire et en "Keramzyt » $\left({ }^{1}\right)$ (la dose à l'intérieur étant supérieure de 44 p. cent).

La figure 2 donne un exemple de spectre obtenu dans un appartement.

L'erreur sur les mesures effectuées par le spectromètre gamma ne dépasse pas \pm 20 p. cent pour la détermination de ${ }^{238} \mathrm{U}$ et \pm 10 p. cent pour ${ }^{232} \mathrm{Th}$ et ${ }^{40} \mathrm{~K}$.

L'erreur sur les mesures effectuées à l'aide de la chambre d'ionisation est de l'ordre de \pm 10 p. cent.

\section{REMERCIEMENTS}

Les auteurs remercient M. Marek Makarewicz pour l'aide apportée en réalisant le programme de calcul du facteur d'accumulation.

\section{BIBLIOGRAPHIE}

[1] Adrijushin N. F., Bulatow B. P., Soviet J. At. Energy, 1965, 19, 1335.

[2] Akkermans J. A., Gamma dose from natural radionuclides in buildings, determined by $\mathrm{Ge} / \mathrm{Li}$-spectrometry. In: Criteria for radiation protection, Condensed Papers, 3 . European congress of the International Radiation Protection Association, Amsterdam, 13-16 May, 1975.

(1) Béton léger avec agrégat artificiel (argile expansé). 
[3] Berger M. J., Dogget J., J. Res. Nat. Bur. Stand., 1956, 56, 89.

[4] Hultovist B., Studies on naturally occuring ionizing radiations. Kgl. Svenska Vetenskaps. Handl., 1956, 6, sér. 4, n 3, 1.

[5] Kiefer H., Koelzer W., Stablein G., Results of measurements relating to the population dose. In: Population dose evaluation and standards for man and his environment, Portorǒz, Yugoslavia, 20-24 May, 1974, Vienne, A.I.E.A., 1974, 305-316.

[6] LEIDMÖRFER M. J. In: Engineering compendium on radiation shielding, (Jaeger R. G., Ed.) Berlin, Springer Verlag, 1968, vol. 1, chap. 44, 233.

[7] OHLSEN H., Bestimmung der mittleren Bevolkerungsbelastung durch naturliche aussere Strahlung auf dem Gebiet der D.D.R. Rapport SZS-14/69, Berlin, 1969.

[8] Peńsko J., Mamont K., Wardaszko T., Measurements of ionizing radiation inside blocks of flats in Poland. Nukleonika, 1969, 14, 415-424.

[9] TAYLOR J. J., Report WAPD RM-217, 1954.

[10] YeAtes D. B., KING B. E., Estimation of the gamma ray natural background radiation dose to an urban population in Western Australia. Health Physics, 1973, 25, 373-379. 